January 21, 2013

Mr. Kevin Ramsey

Senior Project Manager

Fuel Manufacturing Branch

U.S. Nuclear Regulatory Commission

Mailstop: E2C40M

11555 Rockville Pike

Rockville, MD 20852

\title{
SUBJECT: COMPARISON OF RESULTS FOR QUARTER 2 SURFACE WATER SPLIT SAMPLES COLLECTED AT THE NUCLEAR FUEL SERVICES SITE, ERWIN, TENNESSEE \\ DCN: 5198-SR-02-0
}

Dear Mr. Ramsey:

Oak Ridge Associated Universities (ORAU), under the Oak Ridge Institute for Science and Education (ORISE) contract, has completed the collection, sample analysis, and review of split surface water sample results collected at the Nuclear Fuel Services site in Erwin, Tennessee.

Please contact me at 865.574.0685, or Erika Bailey at 865.576 .6659 , if you have any questions.

Sincerely,

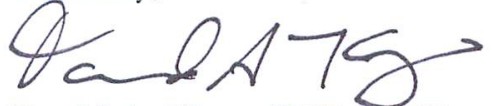

David A. King, CHP, PMP

Sr. Health Physicist/Project Manager

Independent Environmental Assessment

and Verification Program

DAK:fr

electronic distribution:
G. Smith, NRC
M. Chitty, NRC
M. Crespo, NRC
T. Vitkus, ORAU
E. Bailey, ORAU
File/5198

\begin{tabular}{|l|l|}
\hline Distribution approval and concurrence: \\
\hline Technical Review & Initials \\
\hline Laboratory Review & \\
\hline Quality Review & \\
\hline Group Manager Review & \\
\hline
\end{tabular}




\section{COMPARISON OF RESULTS FOR QUARTER 2 SURFACE WATER SPLIT SAMPLES COLLECTED AT THE NUCLEAR FUEL SERVICES SITE ERWIN, TENNESSEE}

Oak Ridge Associated Universities (ORAU), under the Oak Ridge Institute for Science and Education (ORISE) contract, collected split surface water samples with Nuclear Fuel Services (NFS) representatives on November 15, 2012. Representatives from the U.S. Nuclear Regulatory Commission and Tennessee Department of Environment and Conservation were also in attendance. Samples were collected at four surface water stations, as required in the approved Request for Technical Assistance number 11-018. These stations included Nolichucky River upstream (NRU), Nolichucky River downstream (NRD), Martin Creek upstream (MCU), and Martin Creek downstream (MCD).

Both ORAU and NFS performed gross alpha and gross beta analyses, and Table 1 presents the comparison of results using the duplicate error ratio (DER), also known as the normalized absolute difference. A DER $\leq 3$ indicates that, at a 99\% confidence interval, split sample results do not differ significantly when compared to their respective one standard deviation (sigma) uncertainty (ANSI N42.22). The following equation presents the DER calculation.

$$
D E R=\frac{|P-S|}{\sqrt{U_{P}{ }^{2}+U_{S}{ }^{2}}}
$$

Where:

$\mathrm{P}=$ NFS primary sample result

$\mathrm{S}=$ ORAU $\underline{\text { split sample result }}$

$\mathrm{U}_{\mathrm{P}}=$ NFS primary sample one sigma uncertainty

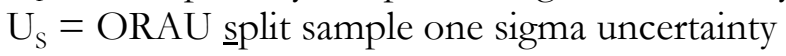

The NFS split sample report does not specify the confidence level of reported uncertainties (NFS 2012). Therefore, standard two sigma reporting is assumed and uncertainty values were divided by 1.96.

In conclusion and as shown in Table 1, all DER values were less than 3 and results are consistent with low (e.g., background) concentrations. 


\section{REFERENCES}

ANSI N42.22. Traceability of Radioactive Sources to NIST and Associated Instrument Quality Control. American National Standards Institute.

NFS 2012. File name "11-15-12 Split Samples.pdf” emailed by Carol Hale/NFS to Jason Lee/ORAU on January 9, 2013. Nuclear Fuel Services. 


\begin{tabular}{|c|c|c|c|c|c|c|c|c|c|c|c|c|}
\hline \multirow[b]{2}{*}{ Quarter } & \multirow[b]{2}{*}{ Station } & \multirow{2}{*}{$\begin{array}{l}\text { ORAU } \\
\text { Sample }\end{array}$} & \multirow{2}{*}{$\begin{array}{c}\text { NFS } \\
\text { Sample }\end{array}$} & \multirow[b]{2}{*}{ Analyte } & \multicolumn{3}{|c|}{ ORAU (pCi/L) } & \multicolumn{3}{|c|}{ NFS (pCi/L) } & \multicolumn{2}{|c|}{ DER } \\
\hline & & & & & Result & Uncert. & MDC & Result & Uncert. & MDC & Value & $\leq 3 ?$ \\
\hline 2 & NRU & 5198W0005 & NRU & Gross beta & 0.79 & 0.23 & 0.75 & 1.77 & 0.64 & 1.92 & 1.4 & YES \\
\hline \multirow[t]{2}{*}{2} & \multirow[t]{2}{*}{ NRD } & \multirow[t]{2}{*}{ 5198W0006 } & \multirow[t]{2}{*}{ NRD } & Gross alpha & 0.05 & 0.11 & 0.39 & 0.65 & 0.45 & 1.49 & 1.3 & YES \\
\hline & & & & Gross beta & 1.14 & 0.24 & 0.76 & 0.41 & 0.43 & 1.53 & 1.5 & YES \\
\hline \multirow[t]{2}{*}{2} & \multirow[t]{2}{*}{$\mathrm{MCU}$} & \multirow[t]{2}{*}{ 5198W0007 } & \multirow[t]{2}{*}{$\mathrm{MCU}$} & Gross alpha & 0.21 & 0.11 & 0.36 & -0.12 & 0.32 & 1.62 & 1.0 & YES \\
\hline & & & & Gross beta & 1.34 & 0.24 & 0.75 & 2.33 & 0.71 & 2.10 & 1.3 & YES \\
\hline \multirow[t]{2}{*}{2} & \multirow[t]{2}{*}{ MCD } & \multirow[t]{2}{*}{ 5198W0008 } & \multirow[t]{2}{*}{ MCD@RR Trestle } & Gross alpha & 0.96 & 0.18 & 0.43 & 1.45 & 0.63 & 1.74 & 0.7 & YES \\
\hline & & & & Gross beta & 1.87 & 0.26 & 0.76 & 2.36 & 0.64 & 1.77 & 0.7 & YES \\
\hline
\end{tabular}

Uncert. $=$ one sigma uncertainty

$\mathrm{MDC}=$ minimum detectable concentration 